\title{
A Performance Comparison of Different Cost Functions for RSS-Based Visible Light Positioning Under the Presence of Reflections
}

\author{
David Plets, Abdülkadir Elyildirim, Sander Bastiaens, Nobby Stevens, Luc Martens, Wout Joseph
}

\begin{abstract}
This paper presents a study of the performance of eight different cost metrics for RSS-based visible light positioning (VLP) under the presence of reflections. A channel model with first-order wall reflections is implemented, after which the distribution of the median ( $\left.\mathbf{p}_{50}\right)$ and maximal errors ( $\left.\mathbf{p}_{\mathbf{9 5}}\right)$ in a typical room configuration are presented and compared for the different metrics. From the simulations, it appears that metrics yielding the lowest median errors correspond to the largest maximal errors, and vice versa. For the $5 \mathrm{~m} \mathrm{x} 5 \mathrm{~m}$ room configuration, median errors range between $6.7 \mathrm{~cm}$ and $8.7 \mathrm{~cm}$, and maximal errors between 11.6 and $25.4 \mathrm{~cm}$. Further, a nearly linear increase in positioning error is observed when the wall reflectance coefficient is increased.

Index Terms - Location, location tracking, localisation, tracking, Visible Light Communication, Visible Light Positioning, VLC, VLP, indoor, photo diode, channel, visible light channel, channel model, channel modelling, channel simulation, reflection, wall, reflectance, algorithm.
\end{abstract}

\section{INTRODUCTION}

Indoor location estimation and tracking systems have gained a huge interest, thanks to the many domains in which they can be applied, e.g., in industrial and office environments, in the healthcare or cultural sector,... One possible application is the guidance of people towards their destination in a building, e.g., in a hospital. Such 'indoor GPS' can be deployed in many different ways: using Ultra-Wide-Band (UWB) timeof-arrival (ToA)-based localisation [1], Angle-of-Arrival (AoA)-based localisation [2], or Received Signal Strength Indicator (RSSI)-based location tracking [3, 4]. Depending on the strictness of the tracking accuracy demands, different options are preferable, where RSSIbased solutions have the lowest installation cost, but also the lowest accuracy. Recently, a relatively cheap, yet accurate alternative to RF-based location tracking has emerged, namely Visible Light Positioning (VLP)based tracking $[5,6]$. Light Emitting Diode (LED) lighting infrastructure is omnipresent nowadays, thanks to its lower power consumption, smaller size, prolonged lifetime compared to conventional lighting technologies.
VLP enables communication via the transmission of modulated light pulses between devices, without compromising the original functionality of illumination. The receiver determines its position by measuring and processing received light intensities with a cheap off-theshelf photo diode (PD). Two main approaches for VLP are currently being investigated: AoA-based VLP and RSSI-based VLP. The former is assumed to have smaller predictions errors but requires a more complex receiver with multiple photo diodes. RSSI-based VLP can work with a single receiving photo diode, and is based on the estimations of the visible light channel attenuations. In areas that are not cluttered, this approach also works well [7], but when the receiver gets near to reflective surfaces, the observed light intensity varies and predictions errors increase. This phenomenon frequently occurs in domains for which VLP technology is considered: offices, supermarkets, and especially industrial warehouses contain many metallic objects that disturb the location estimation. Usually, less attention is given to the metric that is used to process the observed light intensities at the photo diode, although different metrics might exhibit a different positioning performance. In this paper, a framework is presented that (1) implements the visible light channel model for the calculation of light intensities, (2) allows easily switching between different photo diodes and different Lambertian light sources and (3) accounts for diffuse reflections upon walls or objects in the areas. For a variety of eight different location estimation cost metrics, it will be compared how reflections upon walls impair the prediction accuracy and a spatial distribution of the errors will be presented. Further, positioning accuracy will be determined as a function of the reflectance factor of the wall, for three selected metrics. In Section 2, the adopted visible light channel model will be presented, Section 3 will explain the simulation setup. Section 4 discusses the eight approaches to estimate the location, after which results are presented in Section 5. Finally, conclusions of the research and future research tracks are summarized in Section 6. 


\section{CHANNEL MODEL}

In the framework, we account for the Line-of-Sight (LoS) path between transmitting LED source and receiving PD, as well as the first-order reflections via walls or objects. The model parameters of the visible light channel are displayed in Fig. 1. The power $P_{R}$ received at the photo diode is calculated according to the channel model used in [8]:

$$
\mathrm{P}_{\mathrm{R}}=\mathrm{P}_{\mathrm{E}} \cdot\left(\mathrm{h}_{\text {direct }}+\sum_{\mathrm{dA}} \mathrm{h}_{\text {reflected }}^{\mathrm{dA}}\right),
$$

with $\mathrm{P}_{\mathrm{E}}$ the emitted optical power by the LED, $\mathrm{h}_{\mathrm{direct}}$ is the channel gain along along the direct link, and $\mathrm{h}_{\text {reflected }}^{\mathrm{dA}}$ is the channel gain via the first-order reflections on surface element $\mathrm{dA}$. $\mathrm{h}_{\text {direct }}$ can be described as follows:

$$
\mathrm{h}_{\text {direct }}=\mathrm{R}_{\mathrm{E}}(\phi, \gamma) \cdot \frac{\mathrm{A}_{\text {eff }}(\psi)}{\mathrm{d}^{2}} \cdot \mathrm{T}_{\mathrm{R}}(\psi) \cdot \mathrm{G}_{\mathrm{R}}(\psi),
$$

where $\mathrm{R}_{\mathrm{E}}(\phi, \gamma)$ is the radiation pattern of the LED, which is axially symmetric in case of a Lambertian emitter and for order $\mathrm{m}$, reduces to $\frac{\mathrm{m}+1}{2 \pi} \cos ^{\mathrm{m}}(\phi)$, with $\phi$ the angle of irradiance (see Fig. 1). $\mathrm{T}_{\mathrm{R}}(\psi)$ and $\mathrm{G}_{\mathrm{R}}(\psi)$ are the optical filter's gain and the optical concentrator's gain at the receiver, respectively, with $\psi$ the angle of incidence. The field-of-view of the photo diode is two times $\psi_{\mathrm{C}}$ (see Fig. 2 , such that $\mathrm{h}_{\text {direct }}$ becomes equal to zero for $|\psi|>\psi_{\mathrm{C}}$. Within the FOV of the PD, $\mathrm{T}_{\mathrm{R}}(\psi)$ and $\mathrm{G}_{\mathrm{R}}(\psi)$ will be assumed equal to 1 in the following. $\mathrm{d}$ is the distance between the LED and the PD, and $\mathrm{A}_{\text {eff }}$ is the effective photo diode area, which is equal to the photo diode area that is perpendicular to the angle of incidence $\psi$ :

$$
\mathrm{A}_{\mathrm{eff}}(\psi)=\mathrm{A}_{\mathrm{R}} \cdot \cos (\psi),
$$

with $A_{R}$ the actual photo diode area. The first-order reflections will be assumed to be diffuse. As such, each reflecting wall element can be well approximated by a Lambert model with $\mathrm{m}=1$, with $\theta$ the angle of irradiance from the (ideally infinitesimally) small reflecting surface dA. $\rho$ is defined as the surface reflection coefficient, $\theta^{\prime}$ is the angle of incidence on the wall, and $\psi^{\prime}$ the angle of incidence from the reflecting surface onto the PD. The entire channel is assumed to have a flat frequency response.

$\mathrm{h}_{\text {reflected }}^{\mathrm{dA}}=\mathrm{R}_{\mathrm{E}}\left(\phi^{\prime}\right) \cdot \frac{\mathrm{A}_{\mathrm{eff}}\left(\psi^{\prime}\right)}{\mathrm{d}_{1}^{2} \cdot \mathrm{d}_{2}^{2}} \cdot \mathrm{T}_{\mathrm{R}}\left(\psi^{\prime}\right) \cdot \mathrm{G}_{\mathrm{R}}\left(\psi^{\prime}\right) \cdot \rho \cdot \mathrm{dA} \cdot \cos \left(\theta^{\prime}\right) \cdot \frac{\cos (\theta)}{\pi}$.

Again, $\mathrm{h}_{\text {reflected }}$ reduces to zero for for $\left|\psi^{\prime}\right|>\psi_{\mathrm{C}}$ and an axially symmetric LED radiation pattern is assumed $\left(\mathrm{R}_{\mathrm{E}}\left(\phi^{\prime}, \gamma\right)=\mathrm{R}_{\mathrm{E}}\left(\phi^{\prime}\right)\right)$.

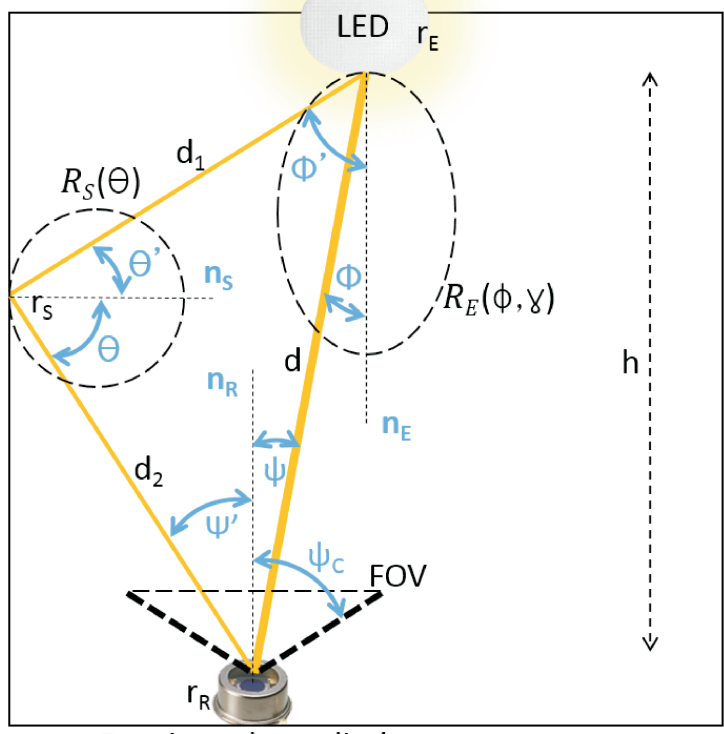

Receiver photo diode

Figure 1: Overview of visible light channel.

\section{SIMULATION CONFIGURATION}

The simulation tests will be executed for the room that is depicted in Fig. 2. The dimensions of the room

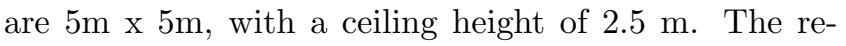
flectance of the wall is denoted as R. Four LEDs with an optical power of $10 \mathrm{~W}$ are attached to the wall at ceiling height, at the locations indicated in Fig. 2. The receiver $\mathrm{PD}$ is assumed to be at a height of $0.85 \mathrm{~m}$. The Lambertian order of the LEDs is equal to $\mathrm{m}=1$, for all four LEDs. In this scenario, we assume that the receiver height is known (e.g., a PD attached to the top of a cart), so the evaluation of the receiver location is reduced to a plane. A receiver grid of $1 \mathrm{~cm}$ will be considered here, meaning that the PD center can be located at $\mathrm{P}=500^{2}$ positions. The 4 walls are divided into 900 elements, i.e., a uniform 30 x 30 grid between the LED height and the PD height, for each of the walls. This means that each wall element has a size of $16.7 \mathrm{x}$ $5.5 \mathrm{~cm}$. Further, we assume that the receiver device is able to demultiplex the contributions of the different LED sources.

The positioning accuracy will first be assessed for a wall reflectance factor $\rho=0.3$ for eight positioning approaches (see Section 4). Secondly, it will be investigated to what extent wall reflections impact the positioning performance, by varying $\rho$ from 0 to 1 . Only first-order reflections will be taken into account, since computing second-order reflections and beyond increases the computation time significantly while their contributions to the received power remain negligible $(<2 \%)[9]$. 


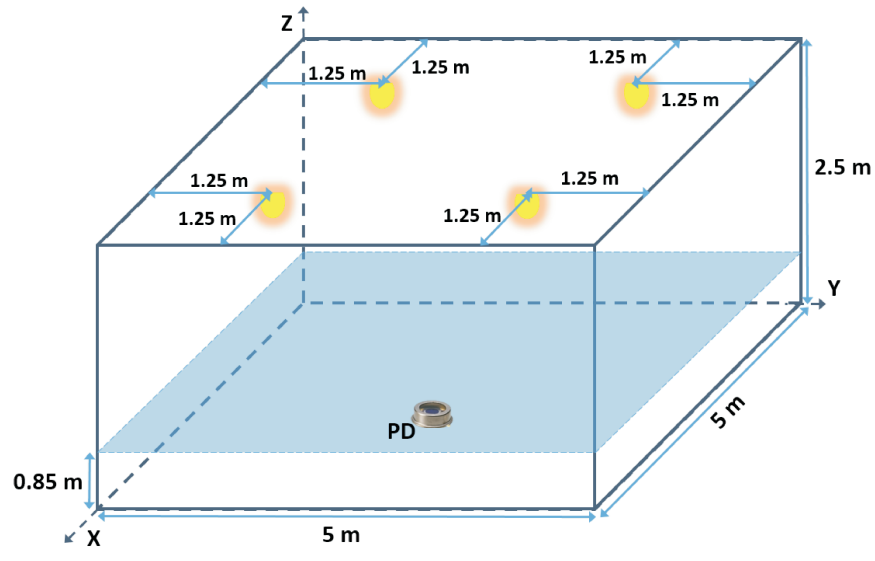

Figure 2: Overview of the simulation setup.

\section{POSITIONING ALGORITHM AND COST METRICS}

The first seven positioning algorithms described hereafter are based on the comparison of the set of so-called measured received photo diode powers $\mathrm{P}_{\mathrm{Ri}}$ from the each $\mathrm{LED}_{\mathrm{i}}(\mathrm{i}=1 . . \mathrm{N})$ at the unknown PD location, with the set of fingerprinted PD powers $\mathrm{P}_{\mathrm{Ri}}^{\mathrm{L} \text {,model }}$ from $\mathrm{LED}_{\mathrm{i}}$ at all locations $\mathrm{L}$ in the grid. For the construction of the fingerprinting database with the $\mathrm{P}_{\mathrm{Ri}}^{\mathrm{L} \text {,model }}$ values, only the direct path is considered. Reflections are not accounted for, since in reality, walls often consist of different parts (wooden door, glass window, concrete or layered wall,...), making it hard to make a correct estimate on the value of the wall reflectance factor $\rho$ and on the expected received reflected power at each location. Further, entering all walls can be a tedious and timeconsuming task in realistic environments. The set of so-called measurements $\left(\mathrm{P}_{\mathrm{R} 1}^{\text {meas }}, \mathrm{P}_{\mathrm{R} 2}^{\text {meas }}, \mathrm{P}_{\mathrm{R} 3}^{\text {meas }}, \ldots, \mathrm{P}_{\mathrm{RN}}^{\text {meas }}\right)$, represent a realistic setup where the direct ray, but also reflected rays are received. In summary, the fingerprinting database only contains the powers along the direct ray from each LED to each $\mathrm{PD}$ location, while the measured values include the direct ray power and the power of all first-order reflections. The larger the reflected power, the larger the differences from the fingerprinting values, and the larger the positioning errors. All power values are calculated according to the channel model presented in Section 2.

The first seven approaches will estimate the unknown location $\mathrm{L}$ to be at the spot where a specific cost function $\mathrm{C}_{\mathrm{X}}^{\mathrm{L}}$ has a minimum, with $\mathrm{X}$ referring to the applied metric. In this paper, the following seven cost metrics are considered:

$$
\mathrm{C}_{\text {square }}^{\mathrm{L}}=\sum_{\mathrm{i}}\left(\mathrm{P}_{\mathrm{Ri}}^{\mathrm{meas}}-\mathrm{P}_{\mathrm{Ri}}^{\mathrm{L}, \text { model }}\right)^{2},
$$

$$
\begin{gathered}
\mathrm{C}_{\log }^{\mathrm{L}}=\sum_{\mathrm{i}}\left(\log \left(\mathrm{P}_{\mathrm{Ri}}^{\text {meas }}\right)-\log \left(\mathrm{P}_{\mathrm{Ri}}^{\mathrm{L}, \text { model }}\right)\right)^{2}, \\
\mathrm{C}_{\text {sqrt }}^{\mathrm{L}}=\sum_{\mathrm{i}}\left(\sqrt{\mathrm{P}_{\mathrm{Ri}}^{\text {meas }}}-\sqrt{\mathrm{P}_{\mathrm{Ri}}^{\mathrm{L}, \text { model }}}\right)^{2}, \\
\mathrm{C}_{\text {inv }}^{\mathrm{L}}=\sum_{\mathrm{i}}\left(\frac{1}{\mathrm{P}_{\mathrm{Ri}}^{\text {meas }}}-\frac{1}{\mathrm{P}_{\mathrm{Ri}}^{\mathrm{L}, \text { model }}}\right)^{2}, \\
\mathrm{C}_{\text {inv_sqrt }}^{\mathrm{L}}=\sum_{\mathrm{i}}\left(\frac{1}{\sqrt{\mathrm{P}_{\mathrm{Ri}}^{\text {meas }}}}-\frac{1}{\sqrt{\mathrm{P}_{\mathrm{Ri}}^{\mathrm{L}, \text { model }}}}\right)^{2}, \\
\mathrm{C}_{\text {abs }}^{\mathrm{L}}=\sum_{\mathrm{i}}\left|\mathrm{P}_{\mathrm{Ri}}^{\text {meas }}-\mathrm{P}_{\mathrm{Ri}}^{\mathrm{L}, \text { model }}\right|^{,} \\
\mathrm{C}_{\text {cubic }}^{\mathrm{L}}=\sum_{\mathrm{i}}\left|\mathrm{P}_{\mathrm{Ri}}^{\text {meas }}-\mathrm{P}_{\mathrm{Ri}}^{\mathrm{L}, \text { model }}\right|^{3},
\end{gathered}
$$

In total, $\mathrm{N}$ x $\mathrm{P}$ values of $\mathrm{P}_{\mathrm{Ri}}^{\mathrm{L}, \text { model }}$ need to be precalculated and stored in a fingerprinting database, i.e., the received power at $\mathrm{P}$ locations from each of the $\mathrm{N}$ LEDs, according to the LoS channel model. This cost function is based on the received power, but a similar approach could be used for e.g. Angle-of-Arrival measurements.

As eighth approach, a slightly different method will also be tested, as presented in [10]. With this approach, measured power values $\mathrm{P}_{\mathrm{Ri}}$ ieas from $\mathrm{LED}_{\mathrm{i}}$ are first reconverted to distances $\mathrm{d}_{\mathrm{i}}^{\text {meas }}$ to $\mathrm{LED}_{\mathrm{i}}$, according the following formula, which has here been extended to random Lambertian modes $\mathrm{m}$ [11].

$$
\mathrm{d}_{\mathrm{i}}^{\text {meas }}=\sqrt[m+3]{\frac{\mathrm{P}_{\mathrm{E}}}{\mathrm{P}_{\mathrm{Ri}}^{\text {meas }}} \frac{\mathrm{m}+1}{2 \pi} \mathrm{h}^{\mathrm{m}+1} \mathrm{~A}_{\mathrm{R}},}
$$

with $\mathrm{h}$ the height difference between LED and PD $(1.65 \mathrm{~m}$ in this study). Here also, calculations are based on only the LoS contribution. Subsequently, the obtained distances $\mathrm{d}_{\mathrm{i}}^{\text {meas }}$ are converted to the most probable location in the receiver plane, according to a standard Least-Square Estimation method. This most probable location corresponds to the location where the following cost function $\mathrm{C}_{\text {dist }}^{\mathrm{L}}$ reaches a minimum.

$$
\mathrm{C}_{\mathrm{dist}}^{\mathrm{L}}=\sum_{\mathrm{i}}\left(\mathrm{d}_{\mathrm{i}}^{\text {meas }}-\mathrm{d}_{\mathrm{i}}^{\mathrm{L}}\right)^{2},
$$

with $\mathrm{d}_{\mathrm{i}}^{\mathrm{L}}$ the real distance between $\mathrm{LED}_{\mathrm{i}}$ and L. Here, $\mathrm{N} \times \mathrm{P}$ values of $\mathrm{d}_{\mathrm{i}}^{\mathrm{L}}$ have to be precalculated and stored, and a conversion to $d_{i}^{\text {meas }}$ is required for each measured power value $\mathrm{P}_{\mathrm{Ri}}^{\text {meas }}$. Moreover, eq. (12) does not straightforwardly allow dealing with non-Lambertian sources or reflections. 


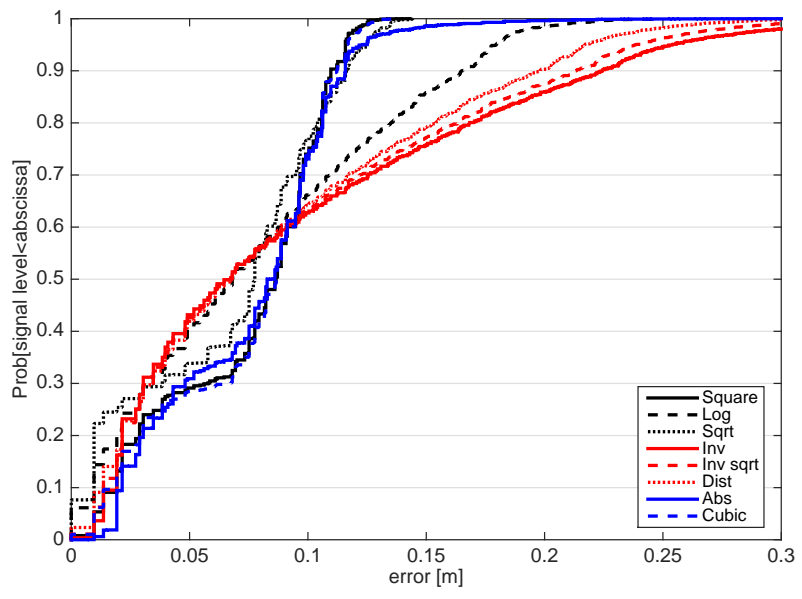

Figure 3: Cumulative distribution function of the positioning errors according to the setup described in Section 3 for the eight metrics defined in Section 4.

Table 1: Median $\left(\mathrm{p}_{50}\right)$ and maximal $\left(\mathrm{p}_{95}\right)$ errors for the eight cost metrics defined in Section 4, applied to the configuration described in Section 3.

\begin{tabular}{|r||r|r|r|r|}
\hline$[\mathbf{c m}]$ & square & sqrt & log & inv \\
\hline \hline $\mathbf{p}_{\mathbf{5 0}}$ & 8.7 & 7.8 & 6.8 & 6.7 \\
\hline $\mathbf{p}_{\mathbf{9 5}}$ & 11.6 & 12.5 & 18.0 & 25.4 \\
\hline \hline$[\mathbf{c m}]$ & inv sqrt & abs & cubic & dist \\
\hline $\mathbf{p}_{\mathbf{5 0}}$ & 6.7 & 8.3 & 8.7 & 6.8 \\
\hline $\mathbf{p}_{\mathbf{9 5}}$ & 23.8 & 12.3 & 11.6 & 21.7 \\
\hline
\end{tabular}

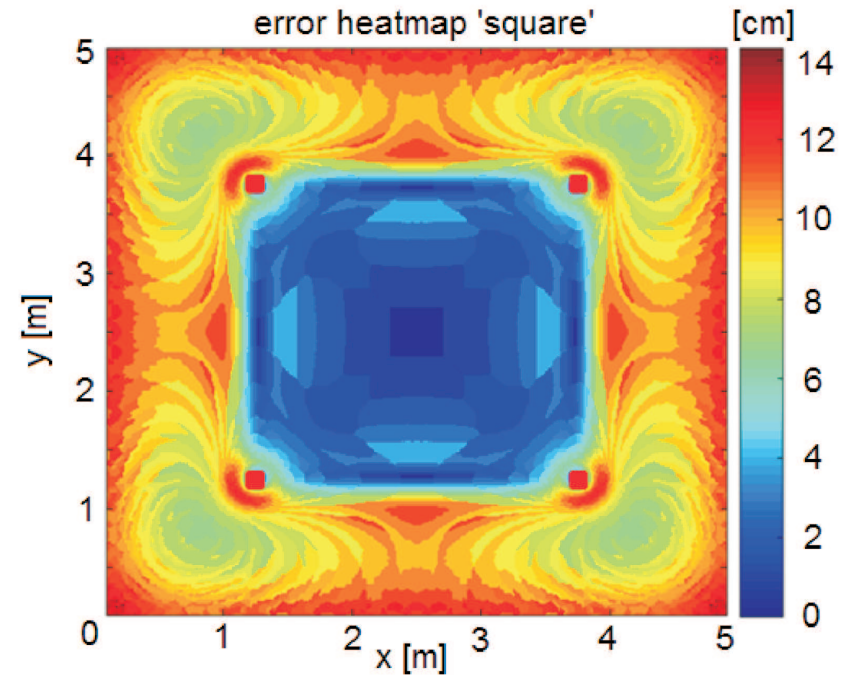

Figure 4: Positioning error due to wall reflections, using the metric 'square'.

\section{RESULTS}

Fig. 3 shows the cumulative distribution function (cdf) of the location estimation error according to the setup described in Section 3 and the metrics defined in Section 4 . The legend in the figure uses the subscripts of the cost functions in Section 4 to denote the metric. Table 1 summarizes the median and maximal errors for all eight presented metrics. Table 1 shows that the lowest median $\left(\mathrm{p}_{50}\right)$ errors are obtained for the metrics 'log', 'inv', 'inv sqrt', and 'dist': 6.7 or $6.8 \mathrm{~cm}$. The lowest maximal $\left(p_{95}\right)$ errors are obtained for the metrics 'square', 'cubic' (both $11.6 \mathrm{~cm})$, 'abs' $(12.3 \mathrm{~cm})$, and 'sqrt' $(12.5 \mathrm{~cm})$. This indicates that the four metrics with the lowest median error, lead to the largest maximal error.

Fig. 4 shows a heatmap of the positioning error due to reflections, using the metric 'square'. In the zone in between the LEDs, errors are mostly limited to $5 \mathrm{~cm}$. Near the walls, errors can increase up to $14 \mathrm{~cm}$. Errors are slightly higher at symmetry lines between the LEDs, since contributions from different LEDs are comparable there, leading to a lower ability to distinguish between adjacent grid locations. In the zone in between the LEDs, errors are mostly limited to $5 \mathrm{~cm}$. Errors are slightly higher at symmetry lines between the LEDs, since contributions from different LEDs are comparable there, leading to a lower ability to distinguish between adjacent grid locations. Figs. 5 and 6 show the heatmap of the positioning error due to reflections, using the metrics 'sqrt' and 'inv sqrt' respectively. For 'sqrt' and 'inv sqrt', the larger errors predominantly occur near the walls (note the different color scale in Fig. 6). For real-life use cases, the receiving PD will most likely be attached on top of a cart (retail), a bed (hospital), a 


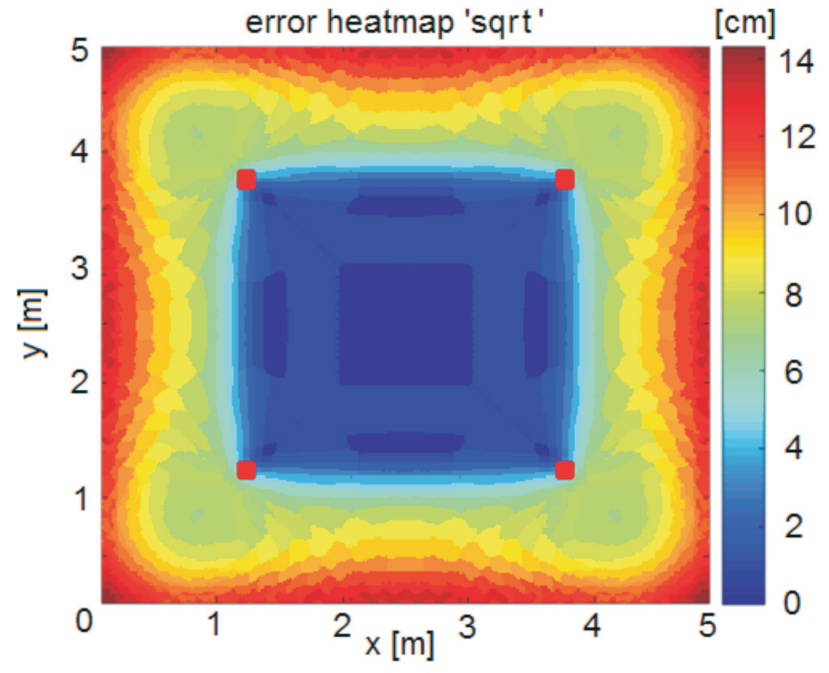

Figure 5: Positioning error due to wall reflections, using the metric 'sqrt'.

forklift truck (industry),... Therefore, in reality, the PD will most likely never be located within e.g., $30 \mathrm{~cm}$ of a wall, making it worth investigating as future work how the positioning errors change when a $30 \mathrm{~cm}$ strip next to the walls is excluded from the analysis.

Further, for three selected metrics (i.e., 'square', 'sqrt', and 'log'), the median and maximal errors are determined as a function of the wall reflectance factor. Results are displayed in Fig. 7 and show that errors exhibit a linear relationship with the reflectance factor $\rho$. The figure shows that when a metric performs better than another one for one reflectance factor, it also performs better for another reflectance factor. The same trend as for $\rho=0.3$ is observed: the metric with the lowest median error (i.e., 'log') has the largest maximal error, for all reflectance factors. Maximal errors can be as high as $55 \mathrm{~cm}$ ('log') for $\rho=1$, but the median error does not exceed $21 \mathrm{~cm}$. For the metric 'square', maximal errors are lower $(38 \mathrm{~cm}$ for $\rho=1)$, but the median error for $\rho=1$ equals $28 \mathrm{~cm}$. Metric 'sqrt' yields errors in between the errors produced by the two metrics 'square' and 'log'.

\section{CONCLUSIONS}

In this paper, the performance of eight different approaches to RSS-based visible light positioning is compared under the presence of reflections. A visible light channel model is built, including first-order reflections. Then, the model is applied to a $5 \mathrm{~m}$ by $5 \mathrm{~m}$ room with four LEDs and the different cost metrics are compared, whereby fingerprinting values only account for the direct ray, whereas measured values also include reflected rays. It is observed that metrics leading to minimal median errors (around $7 \mathrm{~cm}$ ) lead to larger maximal

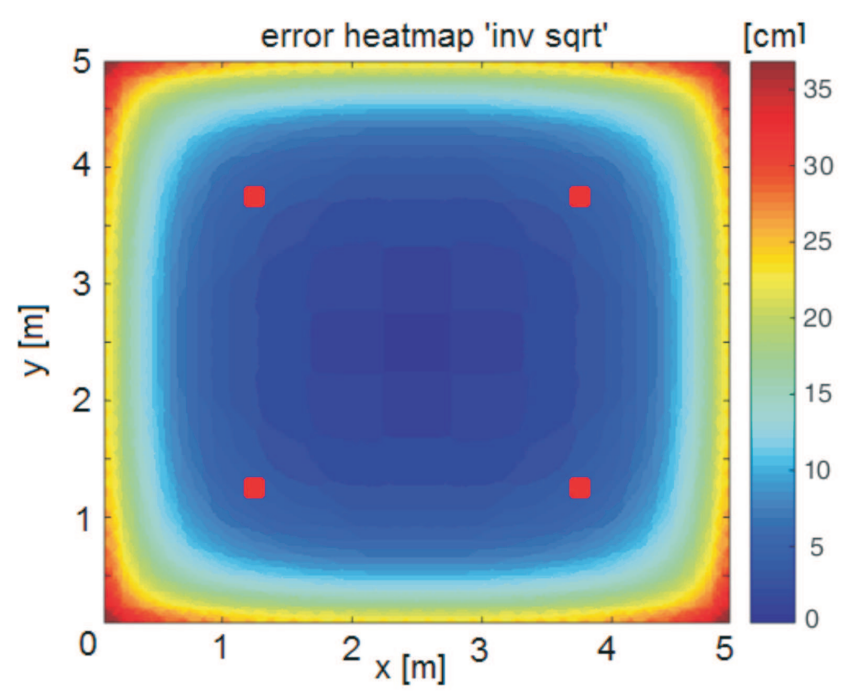

Figure 6: Positioning error due to wall reflections, using the metric 'inv sqrt'.

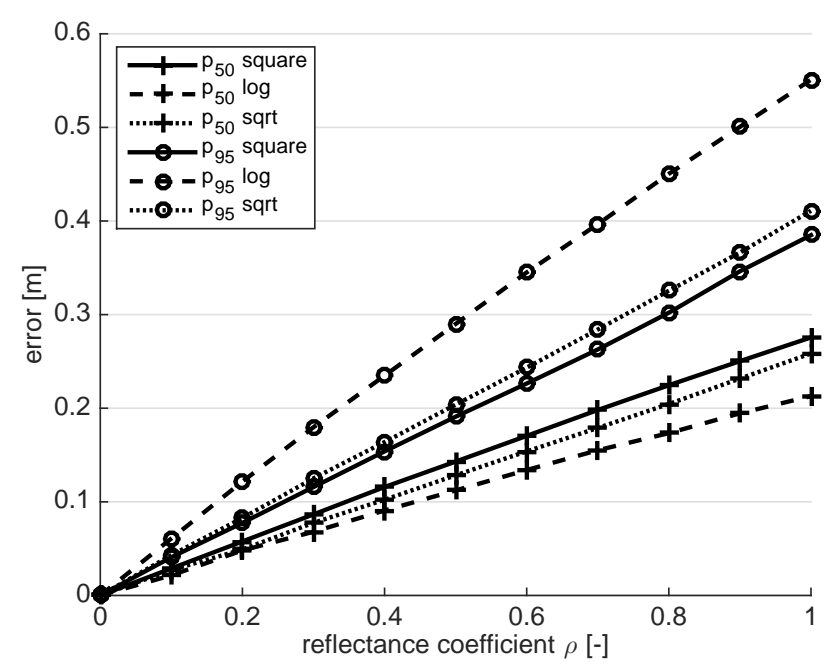

Figure 7: Median ( $\left.\mathrm{p}_{50}\right)$ and maximal ( $\left.\mathrm{p}_{95}\right)$ positioning error as a function of wall reflectance factor $\rho=0$ : 0.1 : 1 under the presence of wall reflections, for five positioning metrics ('square', 'sqrt', and 'log') 
errors (up to around 18 to $22 \mathrm{~cm}$ ). Conversely, metric leading to minimal maximal errors (around $12 \mathrm{~cm}$ ) lead to slight larger median errors (around 8 to $9 \mathrm{~cm}$ ). Different metrics show a different spatial distribution of the error: some metrics yield positioning errors that monotonously increase when moving away from the center of the room (e.g., minimizing squares of deviations between inverse square roots of measurements and fingerprints, 'inv sqrt'), whereas other metrics show a more irregular spatial error distribution (e.g., minimizing squares of deviations between measurements and fingerprints, 'square'). Positioning errors show a linear increase as the wall reflectance factor $\rho$ increases, with median errors between 21 and $28 \mathrm{~cm}$ and maximal errors between 39 and $55 \mathrm{~cm}$, respectively, for $\rho=1$.

Future work consists of including the different noise sources in the simulations, testing larger and more realistic environments, modelling reflective objects that are typically used in industrial environments, and an experimental validation of the simulation results. Further, realistic (non-Lambertian) LED armatures will be modeled, with LED densities and output powers so that realistic illuminance values are obtained in the receiver plane. LED systems with different spectral distributions will be investigated.

\section{Acknowledgment}

This work was executed within LEDsTrack, a research project bringing together academic researchers and industry partners. The LEDsTrack project was co-financed by imec (iMinds) and received project support from Flanders Innovation \& Entrepreneurship.

\section{REFERENCES}

[1] D. Dardari, A. Conti, U. Ferner, A. Giorgetti, and M. Z. Win, "Ranging with ultrawide bandwidth signals in multipath environments," Proceedings of the IEEE, vol. 97, no. 2, pp. 404-426, 2009.

[2] E. Elnahrawy, J. Austen-Francisco, and R. P. Martin, "Adding angle of arrival modality to basic rss location management techniques," in Wireless Pervasive Computing, 200\%. ISWPCŠO\%. 2nd International Symposium on. IEEE, 2007.

[3] J. Trogh, D. Plets, L. Martens, and W. Joseph, "Advanced real-time indoor tracking based on the viterbi algorithm and semantic data," International Journal of Distributed Sensor Networks, vol. 11, no. 10, 2015. [Online]. Available: http://dsn.sagepub.com/content/11/10/271818.abstract

[4] J. Trogh, D. Plets, A. Thielens, L. Martens, and W. Joseph, "Enhanced indoor location tracking through body shadowing compensation," IEEE
Sensors Journal, vol. 16, no. 7, pp. 2105-2114, April 2016.

[5] J. Armstrong, Y. A. Sekercioglu, and A. Neild, "Visible light positioning: A roadmap for international standardization," IEEE Communications Magazine, vol. 51, no. 12, pp. 68-73, December 2013.

[6] A. Jovicic, J. Li, and T. Richardson, "Visible light communication: opportunities, challenges and the path to market," IEEE Communications Magazine, vol. 51, no. 12, pp. 26-32, December 2013.

[7] W. Zhang, M. I. S. Chowdhury, and M. Kavehrad, "Asynchronous indoor positioning system based on visible light communications," Optical Engineering, vol. 53, no. 4, p. 045105, 2014.

[8] T. Komine and M. Nakagawa, "Fundamental analysis for visible-light communication system using led lights," IEEE Trans. Consumer Electronics, vol. 50, pp. 100-107, 2004.

[9] P. Chvojka, S. Zvanovec, P. A. Haigh, and Z. Ghassemlooy, "Channel characteristics of visible light communications within dynamic indoor environment," Journal of Lightwave Technology, vol. 33, no. 9, pp. 1719-1725, May 2015.

[10] S. D. Lausnay, L. D. Strycker, J. P. Goemaere, N. Stevens, and B. Nauwelaers, "A Visible Light Positioning system using Frequency Division Multiple Access with square waves," in 2015 9th International Conference on Signal Processing and Communication Systems (ICSPCS), Cairns, QLD, Australia, 14-16 Dec 2015, pp. 1-7.

[11] W. Xu, J. Wang, H. Shen, H. Zhang, and X. You, "Indoor positioning for multiphotodiode device using visible-light communications," IEEE Photonics Journal, vol. 8, no. 1, pp. 1-11, Feb 2016. 